\title{
LHM Superstrate for High Directivity Microstrip Antenna
}

\author{
Bilal Tütünc $\ddot{u}^{1 *}$, Bülent Urul ${ }^{2}$ \\ ${ }^{1}$ Electronics and Communication Engineering Department, Electric-Electronics Engineering Faculty, Yıldiz \\ Technical University, İstanbul, Turkey \\ ${ }^{2}$ Electronics and Automation Department, Vocational School of Technical Science, Süleyman Demirel University, \\ Isparta, Turkey \\ *bilal1334@gmail.com
}

Received: 28 September 2018

Accepted: 07 February 2019

DOI: $10.18466 /$ cbayarfbe.465379

\begin{abstract}
High antenna directivity is generally desirable for wireless communication systems, whether terrestrial or based on satellites. Most of the solutions proposed for improvement of the directivity of microstrip patch antennas were to design array of several antennas. The particular disadvantage of this method comes from the feeding of each antenna and also from the coupling between each element. A suitable model to eliminate these two disadvantages is to use a separate superstrate structure. For this purpose a Left-Handed medium (LHM) superstrate used and presented in this study. Both the reference antenna and proposed antenna are simulated, fabricated, and tested. The simulation results show good agreement with the measurement results. It is observed that the reference antenna directivity increased by $1.72 \mathrm{~dB}$ with the proposed LHM superstrate according to the measurement results for $12 \mathrm{GHz}$ operating frequency.
\end{abstract}

Keywords: Metamaterial, LHM, Microstrip antennas, Directivity.

\section{Introduction}

In 1968, Vesalago presented that, refractive index may also be negative. He hypothesized that negative refraction can occur if both the electric permittivity $\epsilon$ and the magnetic permeability $\mu$ of a material are negative [1]. In the propagation of electromagnetic waves, the direction of energy flow is given by right-hand rule, but when $\epsilon<0$ and $\mu<0$, the medium is Left-Handed and this materials are called Left-Handed Medium (LHM) or metamaterials. If both the permeability $(\mu)$ and the permittivity $(\epsilon)$ parameters are set negative at the same frequency, an incident electromagnetic wave is inversely refracted and inverse refraction causes focusing. If the electromagnetic wave can be focused instead of transmitting omnidirectional, the directivity and, so the gain will be increased [2].

Different shapes of metamaterial unit cells have been used on the antenna substrate $[3,4]$ on the direct antenna geometry $[5,6]$ or as a superstrate (lens) $[7,8]$ to achieve LHM characteristics in order to improve the antenna performance. In the literature, LHM materials were mostly designed and used for $\mathrm{C}$ and $\mathrm{X}$ band to achieve this improvement. In this study, a LHM structure called symmetrical ring structure (SRS) is designed and produced at $\mathrm{Ku}$ frequency range with $12 \mathrm{GHz}$ operating frequency. Rectangular Microstrip Patch Antenna (MPA) is used as reference antenna and with a few experiments with the help of the CST program, the operating frequency is set to $12 \mathrm{GHz}$. Fabrication and laboratory measurements are made in RF and Microwave
Laboratory in Electronics and Communications department at Yildiz Technical University. Initially results of the reference MPA are obtained without using LHM superstrate. Then a LHM single layer consisting of $2 \times 2$ SRS unit cell is placed at a distance of half wavelength of the MPA as a flat lens and results are obtained. $1.72 \mathrm{~dB}$ increase in the directivity is observed with proposed SRS lens layer according to measurement results.

\section{Materials and Methods \\ 2.1. Design and simulation of reference MPA}

The reference MPA is modeled on CST and then fabricated as being etched through a copper-coated dielectric substrate Rogers RO4350B. The relative dielectric constant is 3.48 , the dielectric loss tangent is 0.0037 and the thickness is $0.762 \mathrm{~mm}$. The width $(\mathrm{W})$ and length $(\mathrm{L})$ of the substrate are taken as twice the size of patch and the thickness of copper is taken $0.035 \mathrm{~mm}$. Patch width (Wp) and patch length (Lp) of the rectangular MPA are calculated by equations given in [9]. For substrate thickness $\mathrm{h}=0.762 \mathrm{~mm}$ and resonance frequency $\mathrm{fr}=12 \mathrm{GHz}$; the patch width $(\mathrm{Wp})$ is calculated as $8.35 \mathrm{~mm}$ and the patch length (Lp) as 6.38 $\mathrm{mm}$. Reference MPA top view and prototype is shown in Figure 1. 


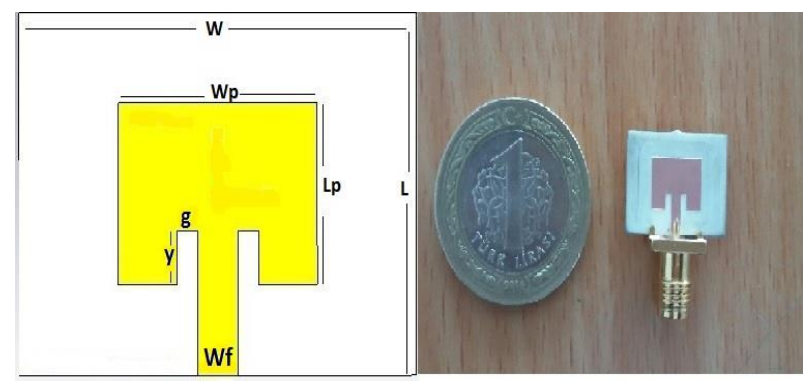

Figure 1. Reference MPA top view.

For impedance matching, two adjacent parallel slits are extended until the desired resonance input impedance value $(50 \Omega)$ is achieved. The feed line extension of the patch " $y$ " is calculated $1.91 \mathrm{~mm}$ and the width of the feed line "wf" is calculated as $1.7 \mathrm{~mm}[10,11]$ and the gap of parallel slit is taken as half of $w f(g=w f / 2=0.85 \mathrm{~mm})$ [9]. S11 curve of the MPA according to the measurement and simulation results are shown in Figure 2.

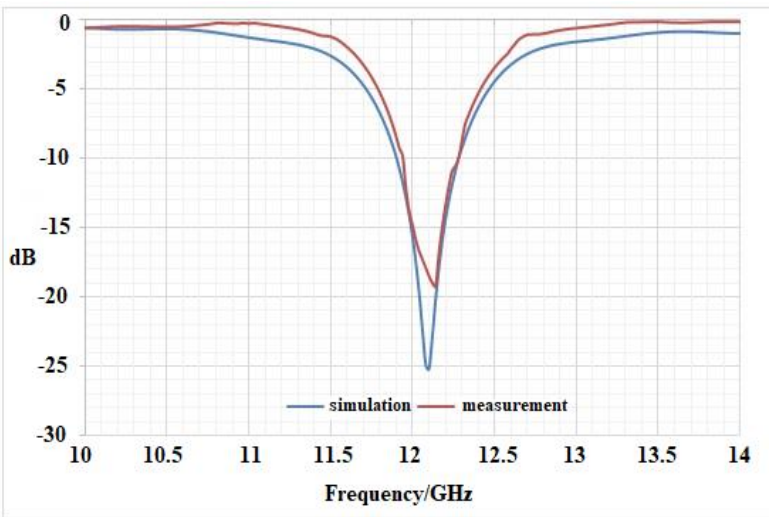

Figure 2. S11 curve of the MPA.

In order to view the increase in the directivity, the simulation and measurement results are taken separately and the far field directivity pattern of MPA without superstrate is drawn for $12 \mathrm{GHz}$. The peak directivity is found as $4.66 \mathrm{dBi} / 4.32 \mathrm{dBi}$ (simulation/ measurement) as shown in Figure 3. Also according to the simulation results Half Power Beam-width (HPBW) is $93^{\circ}$.

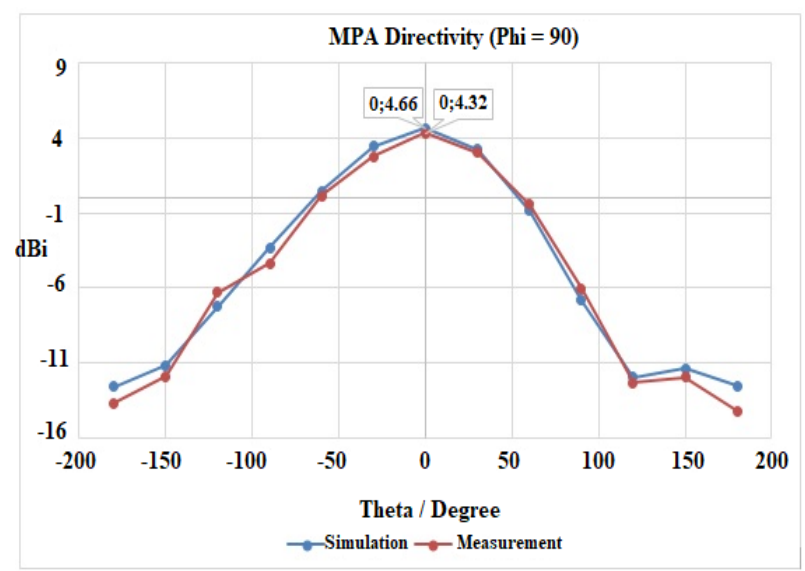

Figure 3. Directivity pattern of the MPA at $12 \mathrm{GHz}$.

\subsection{SRS Unit Cell Design}

This LHM unit cell consists of two symmetric rectangular rings along the $\mathrm{x}$-axis at the front side and a copper conductor strip extending in the y-plane along the substrate at the back side. Also there is a gap in each rings. The same substrate that is used for MPA with predefined characteristics and dimensions is used. With a few optimizations in CST to tune the operating frequency to $12 \mathrm{GHz}$, the dimensions of the unit cell are taken as follows; the length of the long sides of each symmetric rectangular rings $b=5 \mathrm{~mm}$ and the length of the short sides $\mathrm{c}=2.4 \mathrm{~mm}$. The distance between two rings $\mathrm{d}=0.4 \mathrm{~mm}$ and the gap $\mathrm{a}=0.4 \mathrm{~mm}$. The width of the conductive parts $\mathrm{e}=0.5 \mathrm{~mm}$. The length and width of the back strip are $5 \mathrm{~mm}$ and $0.3 \mathrm{~mm}$ respectively. The shape of the designed SRS unit cell structure is shown in Figure 4.

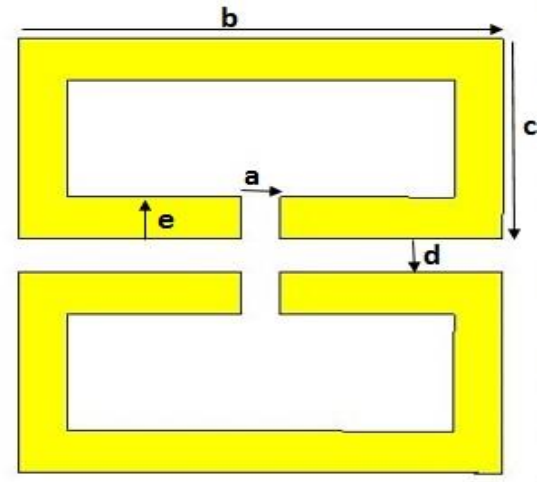

Figure 4. Designed SRS unit cell.

\subsection{Extraction of Media Parameters}

There are several different methods such as NicholsonRoss-Weir (NRW) and Robust Method to obtain the media parameters $(\varepsilon$ and $\mu$ ) from the $S$ parameters $[12,13]$. In this study we chose robust method and obtained the results graphically by MATLAB. $€$ and $\mu$ curves of SRS are shown in Figure 5 and Figure 6. As seen the real values of $\epsilon$ and $\mu$ are negative at $12 \mathrm{GHz}$. ( $\epsilon$ $=-1.6$ and $\mu=-2.1$ ). We investigated whether the SRS is LHM or not at $12 \mathrm{GHz}$, so we have concerned only the transmitted part of the electromagnetic waves. Thus the imaginary parts that represent the "loss energy" are ignored [14]. 




Figure 5. $\epsilon$ curve of SRS.



Figure 6. $\mu$ curve of SRS.

\subsection{SRS as a Slab Periodic Superstrate}

SRS unit cell, whose $\epsilon$ and $\mu$ parameters are confirmed to be negative at $12 \mathrm{GHz}$, have to be converted into a periodic structure to use as a superstrate for a significant increase. Parametric studies is carried out in different dimensions and different quantities for a periodic structure to obtain optimal return loss and optimal radiation parameters and a $2 \times 2$ array at $12 \mathrm{~mm} \times 12 \mathrm{~mm}$ dimensions are noted optimal so the fabrication of SRS superstrate is printed according to this period by LPKF ProtoMat S63 in-house prototyping machine. The top view and bottom view of fabricated SRS superstrate are as shown in Figure 7. This LHM structure can be used as one or more layers [15]. To make a compact design we used only one layer instead of multi-layer structure.



Figure 7. Top and bottom view of fabricated SRS layer

\section{Results and Discussion}

The radiation pattern of the MPA with SRS superstrate is measured by using the free space measurement setup at RF and Microwave Laboratory. Figure 8 and Figure 9 show S11 curve and far field directivity pattern of the proposed LHM superstrate that loaded in front of the patch antenna at a half-wavelength distance $(\lambda 0 / 2=$ $12.49 \mathrm{~mm}$ ) respectively.

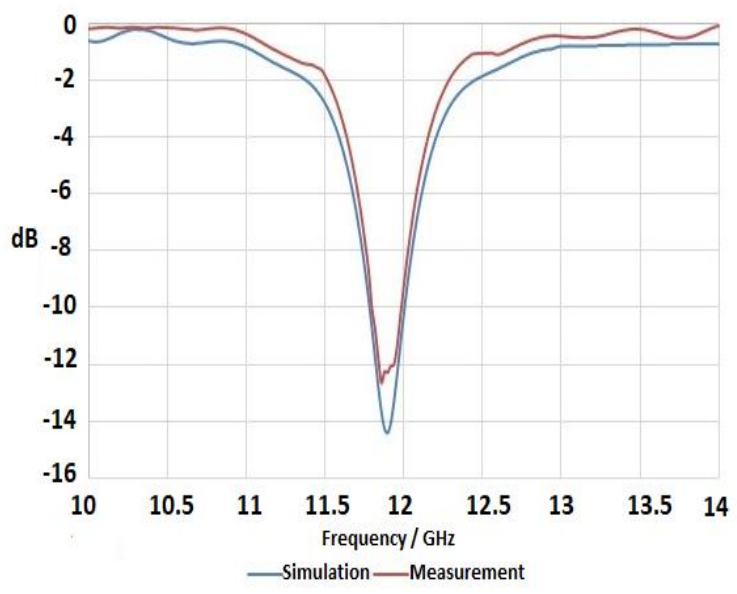

Figure 8. S11 curve of the MPA with SRS.

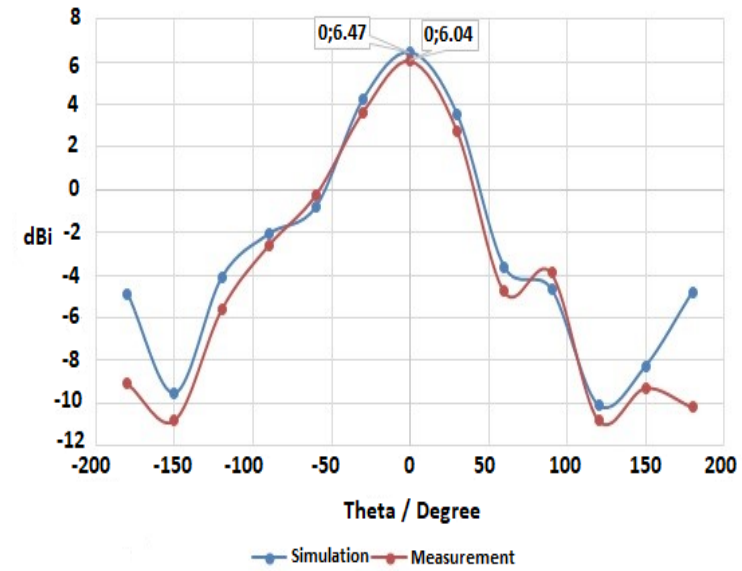

Figure 9. Directivity pattern of MPA with SRS. 
Enhancements on the MPA directivity and changes on HPBW and bandwidth are given in the Table 1. As seen, there is a $1.81 \mathrm{~dB} / 1.72 \mathrm{~dB}$ (simulation / measurement) increase in directivity but as directivity increases, the bandwidth drops as expected. Also the decrease in the HPBW is an evidence of the enhancement of directivity in the radiation pattern. This proposed antenna system is more proper for the studies that requires high antenna directivity instead of wide broadband demands.

Table 1. Changes on directivity, HPBW and bandwidth

\begin{tabular}{|c|c|c|c|c|}
\hline & & $\begin{array}{c}\text { Directivity } \\
\text { dBi }\end{array}$ & $\begin{array}{l}\text { HPBW } \\
\text { Degree }\end{array}$ & $\begin{array}{l}\text { Bandwidth } \\
\text { MHz }\end{array}$ \\
\hline \multirow{2}{*}{$\underline{\Sigma}$} & Sim. & 4.66 & $93^{\circ}$ & 340 \\
\hline & Mea. & 4.32 & & 320 \\
\hline \multirow{2}{*}{$\sum_{\sum}^{+} \frac{\alpha}{n}$} & Sim. & 6.47 & $66^{\circ}$ & 200 \\
\hline & Mea. & 6.04 & & 180 \\
\hline \multirow[b]{2}{*}{ 萬 } & Sim. & $+1.81 \mathrm{~dB}$ & $-27^{\circ}$ & -140 \\
\hline & Mea. & $+1.72 \mathrm{~dB}$ & & -140 \\
\hline
\end{tabular}

\section{Conclusion}

A LHM metamaterial unit cell is proposed and designed as a lens layer for directivity improvement of a MPA at $\mathrm{Ku}$ frequency range with $12 \mathrm{GHz}$ operating frequency. $1.72 \mathrm{~dB}$ increase in the directivity of the reference MPA with proposed SRS lens layer is observed according to measurement results. There is also a reduction in HPBW, which is an evidence of MPA directivity enhancement. The size of metalayers proposed in previous studies are extensive than the patch dimensions of the reference antenna [6-8]. In this study we achieve this improvement with a newly designed metalayer which has a size between the patch and the ground plate dimensions by utilizing same dielectric substrate with the reference antenna.

\section{Author's Contributions}

Bilal Tütüncü: Drafted and wrote the manuscript, performed the experiment and result analysis.

Bülent Urul: Assisted in analytical analysis on the structure, supervised the experiment's progress, result interpretation and helped in manuscript preparation.

\section{Ethics}

There are no ethical issues after the publication of this manuscript.

\section{References}

1. Vesalago, VG. 1968. The electrodynamics of substances with simultaneously negative values of $\epsilon$ and $\mu$. Soviet Physics Uspekhi; 10: $509-514$

2. Pendry, JB. 2000. Negative refraction makes a perfect lens. Physical Review Letters; 85: 3966-3969.
3. Shafique, MF, Qamar, Z, Riaz, L, Saleem, R, Khan, S. 2015. Coupling suppression in densely packed microstrip arrays using metamaterial structure. Microwave and Optical Technology Letters; 57: 759-763.

4. Amanatiadis, S, Karamanos, T, Kantartzis, N. 2017. Radiation efficiency enhancement of graphene $\mathrm{THz}$ antennas utilizing metamaterial substrates. IEEE Antennas and Wireless Propagation Letters; 16: 2054-2057.

5. Dadgarpour, A, Zarghooni, B, Virdee, BS, Denidni, TA. 2015. Millimeter-wave high-gain SIW end-fire bow-tie antenna. IEEE Transactions on Antennas and Propagation; 63(5): 2337-2342.

6. Xiong, H, Hong, J, Tan, M, Li, B. 2013. Compact microstrip antenna with metamaterial for wideband applications. Turkish Journal of Electrical Engineering and Computer Sciences; 21: 2233-2238.

7. Adel, BA, Ahmed, A. 2016. Metamaterial enhances microstrip antenna gain. Microwaves $\& R F ; 7: 46-50$.

8. Li, D, Szabó, Z, Qing, X, Li, EP, Chen, ZN. 2012. A high gain antenna with an optimized metamaterial inspired superstrate. IEEE transactions on antennas and propagation; 60(12): 6018-6023.

9. Balanis, CA. Antenna Theory: Analysis and Design; John Wiley and Sons Press: USA, 1997; pp. 818.

10. Ramesh, M, Yip, KB. 2003. Design formula for inset fed microstrip patch antenna. Journal of Microwaves and Optoelectronics; 3: 510 .

11. İmeci, ST. 2015. E- and H-Shaped High Gain Patch Antennas. Microwave and Optical Technology Letters; 57: 1395-1401.

12. Chen, X, Grzegorcezyk, TM, Wu, BI, Pacheco, J, Kong, JA. 20004. Robust method to retrieve the constitutive effective parameters of metamaterials. Physical Review E; 70: 1-7.

13. Wu, BI, Wang, W, Pacheco, J, Chen, $X$, Grzegorczyk, TM, Kong, JA. 2005. A study of using metamaterials as antenna substrate to enhance gain. Progress In Electromagnetics Research; 51: 295328

14. Çakır, M, Koçkal, NU, Özen, S, Kocakuşak, A, Helhel, S. 2017. Investigation of electromagnetic shielding and absorbing capabilities of cementitious composites with waste metallic chips. Journal of Microwave Power and Electromagnetic Energy; 51: 3142.

15. Bayındır, M, Aydın, K, Özbay, E, Markos, P, Soukoulis, M. 2002. Transmission properties of composite metamaterials in free space. Applied Physics Letters; 81: 120-122. 